\title{
The impact of partial smokefree legislation on health inequalities: Evidence from a survey of I I 50 pubs in North West England
} Karen Tocque ${ }^{1}$, Richard Edwards*2 and Brenda Fullard ${ }^{3}$

\author{
Address: ${ }^{1}$ North West Public Health Observatory, Centre for Public Health, Liverpool John Moores University, North Street, Liverpool, L3 2AY, \\ UK, ${ }^{2}$ Evidence for Population Health Unit, Division of Epidemiology and Health Sciences, Stopford Building, University of Manchester, Oxford \\ Road, Manchester, M13 9PT, UK and ${ }^{3}$ South Sefton Primary Care Trust, 3 rd Floor Burlington House, Crosby Road North, Waterloo, Liverpool L22 \\ OQB, UK \\ Email: Karen Tocque - k.tocque@livjm.ac.uk; Richard Edwards* - MDEASRE2@manchester.ac.uk; Brenda Fullard - brenda.fullard@southsefton- \\ pct.nhs.uk \\ ${ }^{*}$ Corresponding author
}

Published: 0I September 2005

BMC Public Health 2005, 5:9| doi: |0.1 |86/|47|-2458-5-9|
Received: 0I August 2005

Accepted: 0 I September 2005

This article is available from: http://www.biomedcentral.com/|47|-2458/5/9|

(c) 2005 Tocque et al; licensee BioMed Central Ltd.

This is an Open Access article distributed under the terms of the Creative Commons Attribution License (http://creativecommons.org/licenses/by/2.0), which permits unrestricted use, distribution, and reproduction in any medium, provided the original work is properly cited.

\begin{abstract}
Background: The UK government claims that between 10 and $30 \%$ of pubs and bars will be exempt from proposed legislation to achieve smokefree enclosed public places across England. This arises from the contentious inclusion that pubs and bars that do not prepare and serve food and private members clubs, will be able to allow smoking. We aimed to survey pubs and bars across the North West of England to assess smoking policies and the proportion and variations by deprivation level of venues preparing and serving food.
\end{abstract}

Methods: We carried out a telephone survey of II 50 pubs and bars in 14 local authorities across the North West of England. The main data items were current smoking policy, food preparation and serving status, and intention to change food serving and smoking status in the event of implementation of the proposed English partial smokefree legislation.

Results: 29 pubs and bars (2.5\%) were totally smoke-free, 500 (44\%) had partial smoking restrictions, and 615 (54\%) allowed smoking throughout. Venues situated in the most deprived quintiles ( 4 and 5 ) of deprivation were more likely to allow unrestricted smoking (62\% vs $33 \%$ for venues in quintiles I and 2). The proportion of pubs and bars not preparing and serving food on the premises was $44 \%$ (95\% Cl 42 to $46 \%$ ), and ranged from $21 \%$ in pubs and bars in deprivation quintile I to $63 \%$ in quintile 5 .

Conclusion: The proportion of pubs and bars which do not serve food was far higher than the $10-30 \%$ suggested by the UK government. The proportion of pubs allowing unrestricted smoking and of non-food venues was higher in more disadvantaged areas, suggesting that the proposed UK government policy of exempting pubs in England which do not serve food from smokefree legislation will exacerbate inequalities in smoking and health.

\section{Background}

The English Public Health White Paper 'Choosing Health' and the accompanying delivery plan $[1,2]$ state that the government will regulate, with legislation where necessary, to achieve smokefree enclosed public places and workplaces by 2007-2008. However, pubs and bars, 
which do not prepare and serve food and private members clubs, will be able to allow smoking. The UK government claim exempted pubs and bars represent between 10 and $30 \%$ of the total [1].

The Choosing Health proposals raise several concerns. One is that the non-smoking exclusion zone around the bar will be ineffective and harm to the health of staff working in pubs and bars and members clubs will continue. A second is that the effect of smokefree ordinances at reducing smoking prevalence [3] will be undermined by allowing smoking to continue in key social settings like pubs, clubs and bars. Finally, pubs and bars in disadvantaged areas may be less likely to serve food and, because of a higher smoking prevalence among customers, be most likely to stop serving food to allow continued smoking. This may undermine efforts to reduce smoking prevalence in these communities, and perpetuate and exacerbate the already gross inequalities in smoking $[4,5]$ and smoking-related ill health.

Preliminary data from a survey of 29 Local Authorities conducted by the British Medical Association suggest that the $10-30 \%$ figure for exempted premises is an underestimate, particularly outside of the south of England. Thirteen out of 29 (10 located in the north or Midlands) councils estimated that more than $30 \%$ of their pubs did not serve food [6]. Figures from monitoring of pubs by Environmental Health Officers in Northamptonshire found that $54 \%$ of pubs and bars would be exempt, $85 \%$ in Corby, the most deprived Local Authority [7]. Similarly, a recent study of the catering status of 174 pubs on the Local Authority records of Telford and Wrekin borough found that $43 \%$ of pubs would be exempt, $69 \%$ in the most deprived areas [8].

We conducted a survey across 14 Local Authorities in the North West region to assess current smoking policies, the proportion of pubs and bars preparing and serving food, and variations in this proportion by deprivation level of the local area. We hypothesised that pubs and bars from disadvantaged areas would be more likely to allow unrestricted smoking and less likely to prepare and serve food.

\section{Methods}

A structured questionnaire (available online with methodological instructions [9]) was adapted from a pilot survey carried out in Wirral Local Authority and disseminated to all 43 Local Authorities within the North West region. Fourteen Local Authorities agreed to participate: six were predominantly urban, three were predominantly rural and five were mixed urban/rural. Within each Local Authority, a local coordinator identified lists of licensed premises. Either all or a random sample of licensed premises were chosen for inclusion. At least three attempts were made to make contact with the proprietor or owner of chosen establishments. A telephone interview was conducted using the questionnaire except in a few cases where the questionnaire was completed in a face-toface interview.

Some areas focused only on pubs, bars and clubs, whereas others also included hotel and restaurants with licensed bars. The current analysis is largely restricted to pubs and bars and private members clubs, and excludes night clubs, restaurants and hotels. Venues were allocated an areabased deprivation score (Index of Multiple Deprivation (IMD) 2004 [10]) by mapping their postcode to Census Super Output Area which were categorised into deprivation quintiles for all Super Output Areas across the north west.

\section{Results}

There were 1818 licensed pubs, bars included in the sampling frame, of which 1150 pubs and bars $(63 \%)$ agreed to take part, $386(21 \%)$ refused, and $282(16 \%)$ could not be contacted. Of 396 members clubs contacted 195 (49\%) responded and $78(20 \%)$ refused and $123(31 \%)$ could not be contacted. The interviewee was the manager or proprietor for $73 \%$ of pubs and bars participating.

Across the 14 Local Authorities, only 29 pubs and bars $(2.5 \%)$ were totally smoke-free, $500(44 \%)$ had partial smoking restrictions, and 615 (54\%) allowed smoking throughout. Seventy-one per cent allowed smoking at the bar.

The proportion of pubs and bars not preparing and serving food on the premises was 44\% (95\% Confidence Interval (CI) 42 to $46 \%$ ), and ranged from 19 to $55 \%$ across the 13 Local Authorities (one Local Authority excluded because only 14 venues participated). Respondents were asked if they were likely to change their policy on preparing and serving food in response to the English Public Health White Paper proposals. Of pubs and bars that currently served food, $82(13 \%)$ stated that they were likely to stop serving food, and of those that didn't serve food $42(9 \%)$ stated that they were likely to start serving food in response to the proposed English smokefree legislation. The net projected change in the number of venues that would prepare and serve food after introduction of the White Paper proposals was a reduction of $40(3 \%)$, increasing the proportion of non-food pubs from 44 to $47 \%$.

Results stratified by IMD-2004 quintiles are shown in table 1 . The proportion of venues with unrestricted smoking increased with deprivation (Figure 1). In the more affluent areas only $21 \%$ (IMD-2004 quintile 1) and $40 \%$ (quintile 2) allowed smoking throughout compared with 
Table I: Smoking and food preparation policies by IMD-2004 quintiles of deprivation in pubs and bars in I4 Local Authorities in the North West of England.

\begin{tabular}{|c|c|c|c|c|c|c|c|}
\hline & \multirow[t]{2}{*}{ All $(n=1150)$} & \multicolumn{5}{|c|}{ IMD-2004 Quintile } & \multirow{2}{*}{$\begin{array}{c}\text { IMD-2004 } \\
\text { Missing }(n=70)\end{array}$} \\
\hline & & $I(n=70)$ & $2(n=126)$ & $3(n=180)$ & $4(n=355)$ & $5(n=349)$ & \\
\hline$\%$ with unrestricted smoking & 53.8 & 21.4 & 39.7 & 47.2 & 56.3 & 67.1 & 49.3 \\
\hline $\begin{array}{l}\% \text { allowing smoking at the } \\
\text { bar }\end{array}$ & 70.7 & 55.7 & 66.7 & 68.3 & 71.8 & 77.9 & 57.1 \\
\hline $\begin{array}{l}\% \text { currently not preparing } \\
\text { and serving food }\end{array}$ & 43.6 & 21.4 & 28.6 & 38.9 & 36.9 & 63.3 & 40.0 \\
\hline $\begin{array}{l}\text { \% not preparing and serving } \\
\text { food post White Paper } \\
\text { (predicted) }\end{array}$ & 47.0 & 20.0 & 31.8 & 41.1 & 46.2 & 62.8 & 41.4 \\
\hline $\begin{array}{l}\% \text { of food pubs allowing } \\
\text { smoking in food serving } \\
\text { areas }\end{array}$ & $75.7(n=649)$ & $80.0(n=55)$ & $74.4(n=90)$ & $76.4(n=110)$ & $75.0(n=224)$ & $67.2(n=128)$ & $64.3(n=42)$ \\
\hline $\begin{array}{l}\% \text { of pubs allowing children } \\
\text { on premises }\end{array}$ & 60.3 & 77.1 & 79.4 & 69.4 & 52.1 & 54.2 & 57.1 \\
\hline $\begin{array}{l}\% \text { of pubs allowing children } \\
\text { on premises with smoking in } \\
\text { children's areas }\end{array}$ & $81.4(n=693)$ & $85.2(n=54)$ & $83.0(n=100)$ & $81.6(n=125)$ & $75.7(n=185)$ & $78.3(n=189)$ & $75.0(n=40)$ \\
\hline
\end{tabular}

The Index of Multiple Deprivation 2004 (IMD 2004) is a Super Output Area (SOA) level measure of multiple deprivation and is made up of seven SOA level Domain Indices: Income deprivation, Employment deprivation, Health deprivation and disability, Education, skills and training deprivation, Barriers to Housing and Services, Living environment deprivation and Crime. The overall IMD is conceptualised as a weighted area level aggregation of these specific dimensions of deprivation [12].

$56 \%$ in deprivation quintile 4 and $67 \%$ in the most deprived quintile 5 - the absolute difference in proportions between venues in quintiles 1-2 and quintile 4-5 was $28 \%$, (95\% CI 21 to $36 \%)$.

The proportion of pubs and bars not preparing and serving food increased with the level of deprivation (Figure 2) from: $21 \%$ in the most affluent areas (IMD-2004 quintile 1) to $37-39 \%$ in quintiles 3 and 4 , and $63 \%$ in quintile 5 $\left(\chi^{2}=65.1, p<0.001\right)$. Difference in proportions between quintiles 1 and 5 was $42 \%$ (95\% CI $30 \%$ to $51 \%$ ). Based on respondents' stated intentions, the English Public Health White Paper proposals are likely to exaggerate further the gradient in the proportion of non-food pubs and bars by deprivation level (Figure 2).

Over half of the venues allowed children on the premises. This proportion was lowest in venues in the most disadvantaged areas. Around $80 \%$ of pubs and bars where children were allowed permitted smoking in the children's areas. This varied little by IMD-2004 quintile (Table 1). Over three quarters of pubs which prepared and served food allowed smoking in the areas where food was served. This was slightly less in venues in the most deprived quintiles.
Of licensed members clubs, $6 \%$ were smokefree, $33 \%$ had partial smoking restrictions and $61 \%$ allowed unrestricted smoking. There was a gradient in the proportion allowing unrestricted smoking, from 31\% in IMD-2004 quintile 1 to $68 \%$ in quintile 5 .

\section{Discussion}

We found that $44 \%$ of pubs and bars across 14 Local Authorities in the North West of England do not prepare and serve food - far higher than the 10-30\% claimed by the UK government [1]. Furthermore, more respondents indicated that they would stop rather than start serving food in response to the White Paper proposals, resulting in a projected increase of $3 \%$ in the proportion not preparing and serving food.

There was a strong socio-economic gradient in the distribution of non-food serving pubs and bars: with $63 \%$ of businesses located in the most deprived areas (quintiles 4 and 5) not preparing and serving food. Unrestricted smoking was also much more likely in pubs, bars and members clubs in the most deprived areas.

This is the largest and most comprehensive survey of the likely impact of the English smokefree proposals from over 1100 pubs and bars from a wide range of Local 


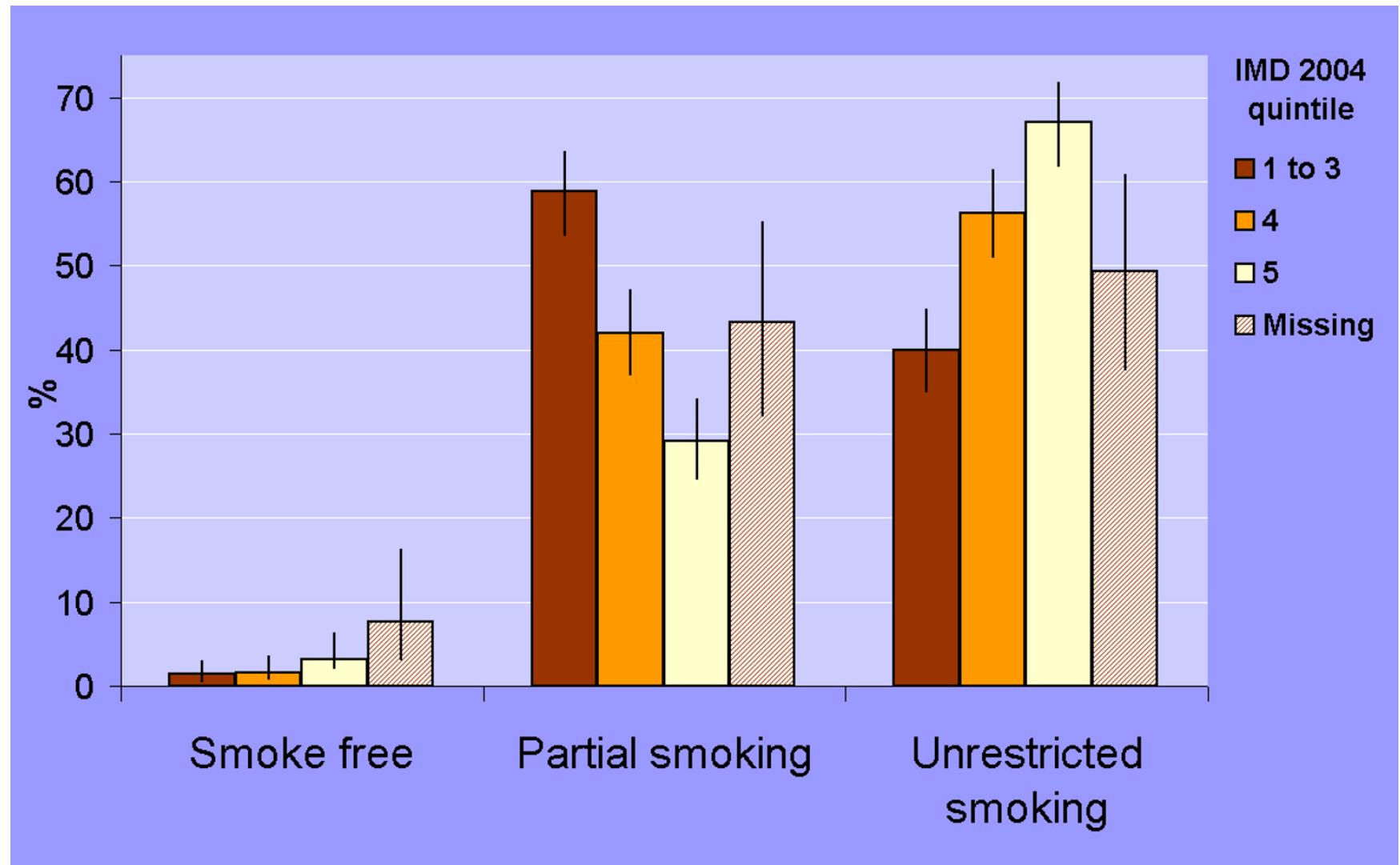

Figure I

Current smoking policies in North West pubs and bars by deprivation quintile (IMD 2004). Data showing percent of venues with $\mathbf{9 5 \%}$ confidence intervals. IMD quintile was allocated by the Census Super Output Area (SOA) of the venue as determined by the postcode of the venue. Quintile I represents the most affluent $20 \%$ of SOAs and quintile 5 represents the most deprived $20 \%$ of SOAs in the North West region.

Authorities across the North West. It explores variations by level of deprivation below Local Authority level of aggregation and is the first to examine likely changes to food serving policy after the proposed legislation is implemented. The findings are based on data collected directly from pubs and bars rather than estimates made by Local Authorities [6].

The White Paper Smokefree Consultation document [11] notes that it has been suggested that the 'Choosing Health' proposals will result in smoking pubs and bars being concentrated in deprived communities, thereby exacerbating health inequalities. This is confirmed by our survey, which found that pubs and bars that don't serve food and hence will be able to allow smoking after 2008 are more concentrated in disadvantaged areas in the North West. It is highly probable that this socio-economic gradient in the location of food/non-food serving establishments will also exist in other parts of England.

\section{Conclusion}

The impact of the 'Choosing Health' proposals are likely to contribute to maintaining the huge inequalities in smoking prevalence and smoking-related morbidity and mortality by perpetuating a strong smoking culture, reducing the impact of cessation in response to smokefree policies, and maximizing exposure of bar staff and nonsmoking customers to passive smoking in the most deprived areas. All these work against the stated Government objective of reducing health inequalities due to smoking.

\section{Competing interests}

The author(s) declare that they have no competing interests. Richard Edwards is unpaid chair of North West ASH and Brenda Fullard is seconded to the North West Public Health Team, Government Office for the North West. 


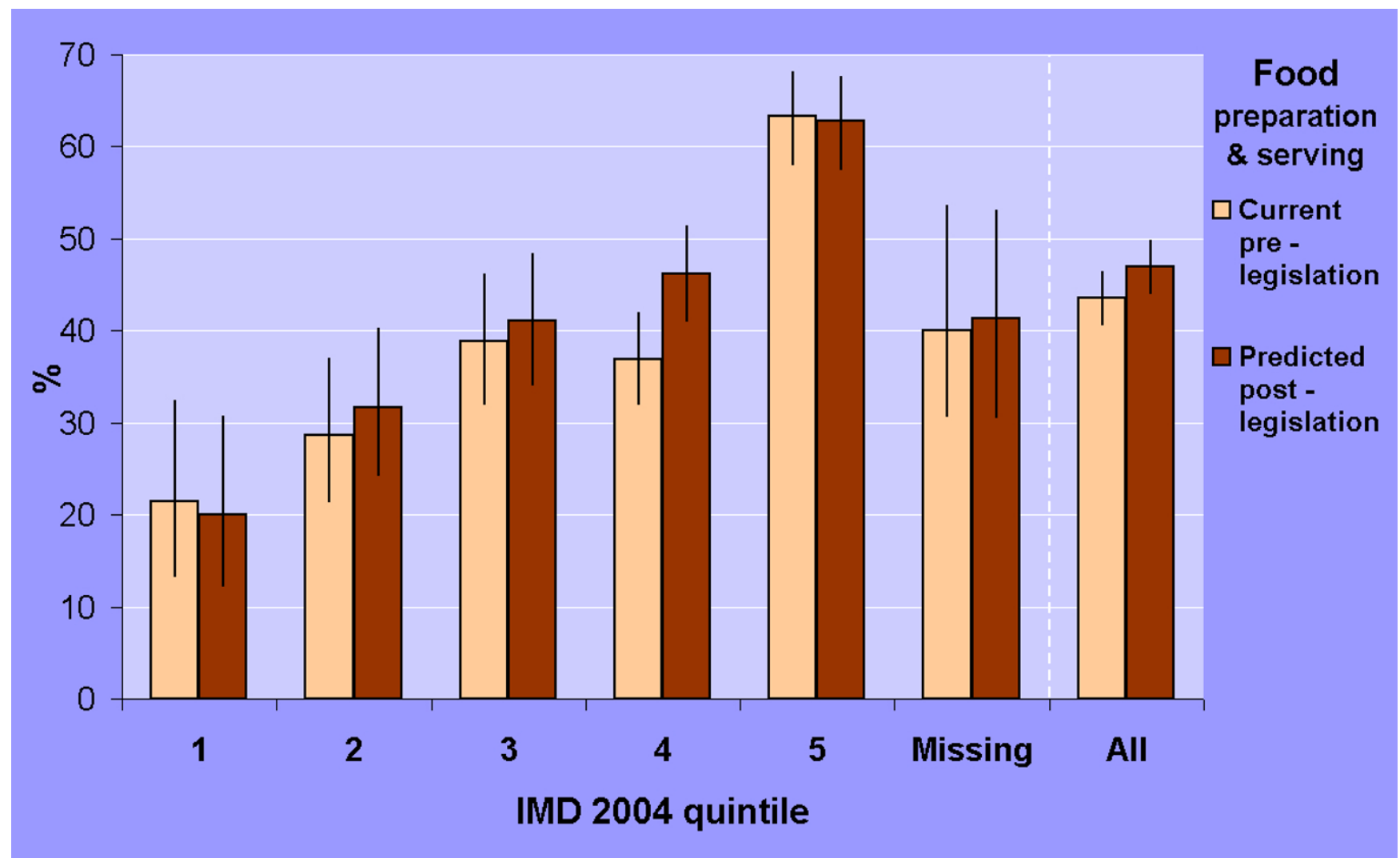

Figure 2

Current and predicted preparation and serving of food in North West pubs and bars by deprivation quintile (IMD 2004). Data showing percent of venues with $95 \%$ confidence intervals. IMD quintile was allocated by the Census Super Output Area (SOA) of the venue as determined by the postcode of the venue. Quintile I represents the most affluent $20 \%$ of SOAs and quintile 5 represents the most deprived $20 \%$ of SOAs in the North West region.

\section{Authors' contributions}

Following a pilot survey carried out by Tina Williams at Smokefree Wirral, KT, RE and BF developed the idea for a regional survey and modified the questionnaire. KT designed the data collection system, co-ordinated the distribution of the questionnaire and collation of the data. Data collection was organised by several local co-ordinators. KT and RE planned and carried out the analysis. The paper was drafted initially by RE, and then developed further with contributions from all the co-authors.

\section{Acknowledgements}

We would like to thank Tina Williams and Smokefree Wirral for a pilot survey and providing the original questionnaire. We are also extremely grateful to all the Local Authority, Primary Care Trust and University staff who helped carry out the survey in the following areas across the North West region: Blackburn with Darwen, Burnley, Bury, Carlisle, Eden, Hyndburn, Liverpool, Manchester, Pendle, Preston, Ribble Valley, Rochdale, Rossendale, and Salford. Particular thanks go to: Richard Holford, Kim Hargreaves,
Claire Probert, Nikolas Storey, Lynne Ratcliffe, Barbara Bellis, Aaron Barker and Mark A Bellis.

The study was carried out and supervised by NHS and University-funded staff. No specific funding was provided for the study from any source. The researchers were independent of the supporting organisation bodies, who had no influence over the design, conduct, analysis or dissemination of the results of the study.

\section{References}

I. Department of Health: Choosing health: making healthier choices easier London, Stationary Office; 2004.

2. Department of Health: Delivering choosing health: making healthier choices easier London, Stationary Office; 2005.

3. Fichtenberg CM, Glantz SA: Effect of smoke-free workplaces on smoking behaviour: systematic review. BMJ 2002, 325: $188-194$

4. Jarvis $M$, Wardle J: Social patterning of individual health behaviours: the case of cigarette smoking. In Social Determinants of Health Edited by: Marmot M and Wilkinson RG. Oxford, Oxford University Press; 1999.

5. Richardson K, Crosier A: Smoking and health inequalities. Action on Smoking and Health, Health Development Agency; 2002. 
6. British Medical Association: Booze, fags and food. 2005 [http:// www.bma.org/ap.nsf/Content/boozefagsandfood]. BMA

7. Charter NSF: Survey data for pubs exempt from smokefree proposals in Choosing Health Northampton, Northampton Primary Care Trust; 2005.

8. Woodall AA, Sandbach EJ, Woodward CM, Aveyard P, Merrington G: The partial smoking ban in licensed establishments and health inequalities in England: modelling study. BMJ 2005.

9. Tobacco Control Research Programme: Centre for Public Health 2005 [http://www.cph.org.uk/tobacco]. Liverpool John Moores University

10. Office of the Deputy Prime Minister: The English Indices of Deprivation 2004: Summary (revised) London, The Stationery Office; 2004.

11. Department of Health: Consultation on the smokefree elements of the Health Improvement and Protection Bill London; 2005.

12. Indices of deprivation 2004: Office of the Deputy Prime Minister [http/ www.odpm.gov.uk/stellent/groups/odpm control/documents/con tentservertemplate/odpm index.hcst? $n=46|0 \&|=3$ ].

\section{Pre-publication history}

The pre-publication history for this paper can be accessed here:

http://www.biomedcentral.com/1471-2458/5/91/prepub

Publish with Biomed Central and every scientist can read your work free of charge

"BioMed Central will be the most significant development for disseminating the results of biomedical research in our lifetime. "

Sir Paul Nurse, Cancer Research UK

Your research papers will be:

- available free of charge to the entire biomedical community

- peer reviewed and published immediately upon acceptance

- cited in PubMed and archived on PubMed Central

- yours - you keep the copyright

Submit your manuscript here:

http://www.biomedcentral.com/info/publishing_adv.asp 